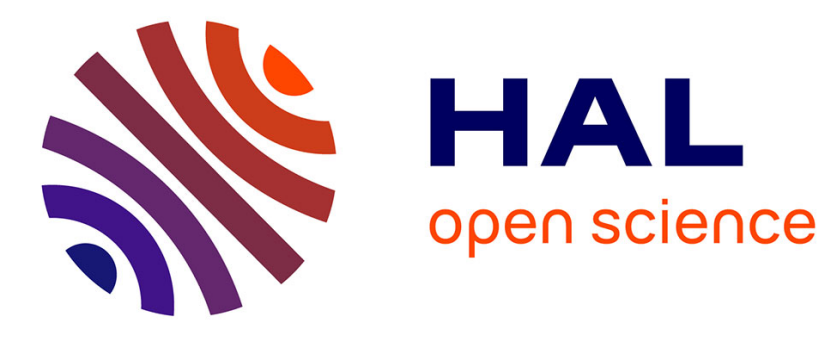

\title{
La typification des atteintes aux bonnes moeurs
}

\author{
Baudouin Dupret
}

\section{To cite this version:}

Baudouin Dupret. La typification des atteintes aux bonnes moeurs: Approche praxéologique d'une affaire égyptienne. International Journal for the Semiotics of Law / Revue Internationale de Sémiotique Juridique, 1998, 33, pp.303-322. halshs-00179362

\section{HAL Id: halshs-00179362 \\ https://shs.hal.science/halshs-00179362}

Submitted on 15 Oct 2007

HAL is a multi-disciplinary open access archive for the deposit and dissemination of scientific research documents, whether they are published or not. The documents may come from teaching and research institutions in France or abroad, or from public or private research centers.
L'archive ouverte pluridisciplinaire HAL, est destinée au dépôt et à la diffusion de documents scientifiques de niveau recherche, publiés ou non, émanant des établissements d'enseignement et de recherche français ou étrangers, des laboratoires publics ou privés. 
Revue Internationale de Sémiotique Juridique Vol.XI no.33 [1998]

\section{LA TYPIFICATION DES ATTEINTES AUX BONNES MOEURS Approche praxéologique d'une affaire égyptienne}

par

\section{BAUDOUIN DUPRET}

CNRS / CEDEJ, Le Caire

Travaillant sur les usages de la référence islamique dans les pratiques juridiques et judiciaires contemporaines en Egypte, j'ai été frappé par la nature extrêmement peu technique des représentations que les professionnels du droit pouvaient avoir de la sharî́a (Loi islamique). Il m'a semblé que ces représentations ne se démarquaient pas - ou fort peu - des représentations propres au sens commun. Le contexte égyptien est par ailleurs très largement déterminé par la question de la moralité publique et de sa définition. On peut même penser que le propre de la sharî‘a - ce que j'appelle le répertoire juridique islamique - n'est pas tant de renvoyer à un système juridique particulier que d'imposer l'inscription du débat dans un cadre moral. C'est dès lors assez naturellement que surgit la question des modes constitutifs des sens professionnel et profane du droit et, plus particulièrement, de la moralité publique et de sa traduction juridique. Je voudrais, dans cet article, tenter de décrypter certains des mécanismes qui permettent d'expliquer les manières d'appréhender, au niveau aussi bien du sens commun que du sens judiciaire, le droit et la justice.

La psychologie cognitive étudie le processus de catégorisation et la théorie juridique s'intéresse à l'opération de qualification. L'usage de deux termes différents (catégorisation et qualification) inciterait à établir une dichotomie entre les opérations cognitives propres au sens commun et les procédures techniques liées au travail professionnel. Pourtant, les sens ordinaire et judiciaire ne peuvent être complètement dissociés, de la même façon que le sociologue, pour reprendre le langage ethnométhodologique, ne peut prétendre à une position en "surplomb" de la réalité sociale, du sens commun la décrivant et des pratiques l'habitant, toutes choses qu'il regarderait de manière "ironique". Plutôt donc que de considérer les deux opérations séparément, je propose d'utiliser un même outil 
analytique: la typification. D'un point de vue sémiotique, la typification consiste dans le rapprochement de situations factuelles avec une image collective (par exemple, celle de porter atteinte à la pudeur). La typification est donc cette opération par laquelle l'individu, qui dispose d'une collection de paradigmes, situe et évalue par rapport à ceux-ci les différentes situations de la vie quotidienne. Cette conception de la typification pose toutefois le problème de la surévaluation de la teneur épistémique des catégories typiques. Ce problème semble pouvoir être surmonté par l'adoption d'une

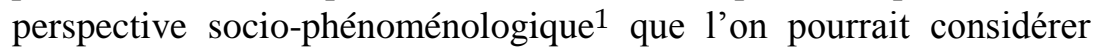
comme plus praxéologique. En ce sens, la typification ne serait plus l'attribution à une situation des qualités lui permettant de relever d'une catégorie (opération prédicative), mais l'opération d'attribution elle-même, la formule des opérations possibles face à une situation donnée. Cette approche par la typification devrait permettre à la fois de réduire l'opposition des sens ordinaire et judiciaire et de prendre la mesure de ce que la pragmatique propre aux différentes activités ordinaires et professionnelles implique comme différentiation dans la construction du sens. En d'autres mots, il devrait être possible de ne plus opposer les sens ordinaire et judiciaire sur le plan sémantique tout en les distinguant sur le plan pragmatique.

Dans cet article, qu'il faut situer dans le cadre plus large d'une recherche en cours sur les figurations de la moralité judiciaire en Egypte, je voudrais procéder en deux temps. Il s'agira d'abord de revenir sur les développements de la psychologie cognitive en matière de catégorisation et sur ceux de la théorie juridique de la qualification. Dans un deuxième temps, on visera à démontrer qu'il n'y a pas lieu d'en déduire l'existence d'une dichotomie radicale entre sens commun du droit et savoir technique juridique, leur différenciation tenant davantage à la question de leur pragmatique qu'à celle de leur sémantique. On illustrera le propos à l'aide d'un matériau tiré d'une affaire de viol collectif survenue en janvier 1985, au Caire.

1 S‘inspirant de la philosophie de Husserl et des développements sociologiques que lui ont donnés Schutz et, à la suite de Garfinkel, l'ethnométhodologie. 


\section{Catégorisation et qualification}

\subsection{Psychologie cognitive et catégorisation}

Pour la psychologie cognitive, l'individu, confronté à la multiplicité et à la complexité de la réalité, procède à des opérations de limitation et de réduction en organisant cette réalité dans des catégories. La question est alors de savoir comment nous découpons la réalité. Schématiquement, il s'agirait d'introduire des continuités et des discontinuités, des regroupements et des distinctions. Catégoriser consiste à subsumer un objet sous une classe d'appartenance, laquelle est prise pour naturelle. Le cadre d'analyse est ainsi représentationnaliste: l'individu juxtapose ou compare l'exemplaire à catégoriser et un type, ce que Jackendoff appelle un token et un type. ${ }^{2}$ Proposant une grammaire universelle des concepts, cet auteur tente de montrer que nous comprenons le monde à travers des exemples idéaux et également des zones grises sensibles au contexte qui entourent ces idéaux. ${ }^{3}$ S'attachant plus précisément à la vision, Jackendoff nous parle d'une grammaire mentale incorporant un "vocabulaire visuel" et, plus encore, un jeu de catégories visuelles stéréotypiques. Si l'occurrence comporte tous les critères du stéréotype, la catégorisation opèrera indépendamment du contexte, alors qu'une approximation dans la satisfaction des critères fera entrer le contexte dans l'opération catégorisante.

Jackson tire argument de cette théorie de la catégorisation pour développer sa thèse de la narrativité. Il entend par narratif, "non pas le récit oral (ou écrit) d'une histoire, mais bien la construction du sens en termes de séquences d'événements et d'actions". 4 Cette question de la nature narrative de la catégorisation mérite qu'on s'y attarde, dans la mesure o elle fonde certains développements sur la

2 R. Jackendoff, Consciousness and the Computational Mind (Cambridge, MA: MIT Press, 1987).

3 R. Jackendoff, Patterns in the Mind. Language and Human Nature (Hemel Hempstead: Harvester Wheatsheaf, 1993).

4 B.S. Jackson, Making Sense in Law. Linguistic, Psychological and Semiotic Perspectives (Liverpool: Deborah Charles Publications, 1995), 227. 
typification. Jerome Bruner ${ }^{5}$ considère l'existence d'une forme de compétence narrative comme innée. Pour être mise en oeuvre, quatre composantes grammaticales doivent être réunises: le moyen de mettre l'accent sur l'action humaine ("agentivité", c'est-à-dire le fait pour l'action d'être dirigée vers des buts contrôlés par des agents); l'établissement et le maintien d'un ordre séquentiel (les événements et les états doivent être "linéarisés" de manière standard); une sensibilité à ce qui, dans l'interaction humaine, est canonique ou non; l'approximation d'une perspective de narrateur (on ne peut être "sans voix"). ${ }^{6}$ La narrativité constitue, dans cette perspective, le principe organisateur de ce que Bruner appelle la "psychologie populaire" (folk psychology). Si le terme me paraît particulièrement maladroit, il n'en recouvre pas moins l'idée parfaitement acceptable d'un ensemble socialement partagé de "descriptions plus ou moins interconnectées et plus ou moins normatives de la façon dont les êtres humains "fonctionnent", de ce à quoi ressemblent notre esprit et celui des autres, de ce à quoi on peut légitimement penser qu'une action située ressemblera, des modes de vie possibles, de la façon dont on peut s'engager et ainsi de suite". ${ }^{7}$ En tout cela sont comprises les règles situationnelles de l'agir et du parler (ce que Jackson rapporte entre autres au principe coopératif de Grice $\left.{ }^{8}\right)$. Schémas et scripts deviennent des concepts-clés. Ainsi, Canter parle de "schémas d'actions attendues et organisées, souvent lâchement, autour d'objectifs personnels",, l'idée centrale étant que le sens est construit au départ de notre "reconnaissance de la connectibilité des événements". 10.

Deux théories s'opposent sur la question de l'appartenance à une catégorie. D'une part, la théorie aristotélicienne classique pour

5 J. Bruner, Acts of Meaning (Harvard: Harvard University Press, 1990).

6 Ibid., à 77-79.

7 Ibid., à 35 .

8 "Il existe des conventions sociales qui à la fois contraignent notre comportement conversationnel et nous aident à communiquer nos intentions" (texte non publié; cf. Jackson, supra n.4, à 70).

9 D. Canter, Criminal Shadows. Inside the Mind of the Serial Killer (London: Harper Collins, 1994), 228.

10 Ibid. 
laquelle l'appartenance est fonction de la présence d'un ensemble de propriétés considérées comme nécessaires et suffisantes). D'autre part, la théorie probabiliste pour laquelle l'appartenance est fonction d'une similitude avec un exemplaire typique de la catégorie (prototype) ou d'un air de famille. Pour cette dernière, le jugement d'appartenance est formé par la comparaison des traits d'un objet et de son prototype ou de sa famille, de sorte que cet objet paraisse aller ensemble avec les autres objets de la catégorie, que sa similarité à eux soit plus forte que celle qu'il peut avoir avec des objets extérieurs à la catégorie. "Cette théorie du prototype a servi à rejeter la conception dite classique de la catégorisation: on ne décide pas si un objet appartient à une catégorie en examinant s'il présente chacune des propriétés nécessaires et suffisantes spécifiées par la définition de la catégorie, mais en le comparant à des membres typiques de la catégorie, à des exemples standards ou à des propriétés typiques". ${ }^{11}$ Dans cette perspective, la catégorisation d'un objet concret se fait à un certain niveau (niveau de base), celui à partir duquel on peut inférer un maximum de propriétés susceptibles de le déterminer. ${ }^{12}$ Quéré fait parfaitement remarquer que la catégorisation a pour cadre, dans cette approche psychologique, une théorie représentationnaliste de l'esprit: "toute catégorisation est supposée mettre en jeu une représentation mentale des catégories auxquelles on décide d'affilier tel ou tel objet". ${ }^{13}$ Si la théorie probabiliste abandonne l'idée d'une définition en termes de conditions nécessaires et suffisantes, elle reste cependant une version de la théorie descriptive de la référence.

A parcourir le traitement médiatique des circonstances et du jugement de l'affaire de "la jeune fille de Ma'âdî", ce procès de six jeunes gens, de 18 à 30 ans, accusés d'avoir enlevé, séquestré et volé une jeune femme et son fiancé et d'avoir violé la première, on ne manque pas de tomber sur une série d'images que l'on qualifierait volontiers, dans une perspective de psychologie cognitive, de paradigmatiques de la pudeur féminine, de la sexualité, de son

11 L. Quéré, "Présentation", in B. Fradin, L. Quéré et J. Widmer (sous la dir.), L'enquête sur les catégories (Paris: Ed. de l'EHESS, 1994), 13-14 (Raisons pratiques 5).

12 E. Rosch, "Principles of Categorization", in E. Rosch and B.B. Lloyd, eds., Cognition and Categorization (Hillsdale: L. Erlbaum, 1978).

13 Quéré, supra n.11, à 14. 
contrôle et de la répression de la transgression. Qu'il s'agisse de l'appellation générale de cette affaire de mœurs, qadiyyat fatât alMa âdî (l'affaire de la jeune fille de Ma'âdî), ou de certains titres et sous-titres de la presse, l'action semble toujours catégorisée par son rattachement à un archétype. Il s'agit ici de la catégorie de "crime répugnant" (jarîma bashi a). Celle-ci semble regrouper, dans la presse, tous les types d'affaires de moeurs, de la pornographie à la prostitution et au viol. La présentation de l'affaire de Ma'âdî la fait tomber sous l'évidence de cette appartenance catégoriale. On trouve ainsi en titre cette déclaration de la jeune fille: "J'ai fait face à des monstres qui m'ont déchiré la peau de leurs crocs" (wajahtu wuhûshan mazaqû admiyatî bi-anyâbihim). Le traitement médiatique d'une autre affaire de viol, l'affaire de "la jeune fille de 'Ataba" (qadiyyat fatât 'Ataba), confirme ce fonctionnement paradigmatique. Il est ainsi question d'une jeune fille qui, un soir du "mois béni de Ramadan", a fait l'objet d'une "agression répugnante". On peut noter que, parmi les éléments constitutifs du paradigme "crime répugnant", la question de la virginité occupe une place prépondérante. Le fait par exemple qu'aux dires du médecin légiste, la jeune femme de Ma'âdî soit demeurée vierge, en dépit de l'agression sexuelle collective dont elle a fait l'objet, constitue de toute évidence un problème, dans l'opération de catégorisation des journalistes, que seule une explication médicale circonstanciée a pu compenser. On notera simplement que, conjointement et symétriquement au paradigme de "crime répugnant", est apparu celui de "relations sexuelles licites" - ce faisant, on anticipe une des questions particulièrement bien traitées par l'ethnométhodologie et l'analyse de conversation (cf. infra). La catégorisation d'une occurrence en "crime répugnant" passe ici par sa constitution en négatif de la catégorie "relations sexuelles licites".

Les catégorisations spontanées des relations sexuelles que des magistrats, hors de leur cadre professionnel, m'ont proposées permettent également d'illustrer cette approche représentationnaliste par le paradigme et ses occurrences. Pour les différents magistrats, tous de sexe masculin, avec qui je me suis entretenu, la catégorie "relations sexuelles licites", c'est-à-dire inscrites dans le cadre légal du mariage, constitue la référence de base pour l'évaluation de tout acte sexuel. La contrainte n'apparaît pas prépondérante, de sorte que l'idée de viol est exclue à l'intérieur d'un couple légalement marié. 
Comme le paradigme "relations sexuelles licites" implique les relations d'un homme et d'une femme dans le cadre du mariage par toute intromission d'un organe de l'homme ou d'un objet qu'il tient dans le sexe de la femme, le viol, qui est l'accomplissement de relations sexuelles illicites par contrainte, suppose nécessairement un homme et une femme et la relation décrite plus haut. Cette catégorisation du viol exclut dès lors toute inversion de rôle des protagonistes (une femme ne peut pas violer un homme), toute relation qui ne serait pas strictement celle décrite plus haut (comme la sodomie) et toute situation homosexuelle (un homme ne peut pas violer un homme).

Pris du point de vue de la psychologie, le sens commun a vu dans les incidents de Ma'âdî la réunion des conditions nécessaires à leur catégorisation de viol. Ainsi, si l'on s'en tient à l'analyse de Rosch, on serait tenté de dire qu'un niveau de base des catégories "acte répugnant" et "relations sexuelles licites" peut être décelé, à partir duquel tout un complexe de propriétés ont été inférées qui permettaient de déterminer les faits qui ont été rapportés. On peut se tourner à présent vers le traitement que le droit opère de ces mêmes faits et, plus généralement, vers l'opération juridique de qualification.

\subsection{Théorie juridique et qualification}

Tout système juridique semble devoir se caractériser par l'adoption d'un système classificatoire ou catégoriel propre: "le droit est un processus, sans cesse renouvelé, de classification du réel". ${ }^{14}$ Confrontés à un objet, les individus, par leur interaction, sont conduits à étiqueter, typifier, qualifier cet objet, et toute nouvelle confrontation est source d'ajustement des schèmes catégoriels et classificatoires existants. Ceci ne se manifeste jamais avec autant d'évidence que dans la fonction de juger.

Si l'on tente de schématiser l'évolution de cette dernière dans l'espace occidental, on perçoit qu'elle se produit sous le signe de la perpétuation d'un idéal systématique et logicien. ${ }^{15}$ Le "fétichisme de

14 L. Assier-Andrieu, "Le juridique des anthropologues" Droit et Société 5 (1987), 89-107.

15 J. Lenoble et F. Ost, Droit, mythe et raison. Essai sur la dérive mythologique de la rationalité juridique (Bruxelles: Publications des Facultés universitaires Saint-Louis, 1980). 
la loi écrite et codifiée" en est la parfaite illustration. L'opération de juger n'est plus alors qu'une mécanique répondant aux "seuls schèmes de la logique hypothético-déductive". ${ }^{16}$ Entre l'Ancien et le Nouveau Régime, on peut parler d'une "perpétuation modalisée" de l'idéal logicien, ne serait-ce qu'au niveau de l'interprétation proprement dite. ${ }^{17} \mathrm{Ce}$ sont en effet toujours les mêmes présupposés de complétude, de cohérence et d'unité de sens qui continuent à dominer ce travail et sont maintenant transposés à l'ensemble du système de droit. "Au-delà de l'alternative réductrice de l'inféodation du discours juridique à l'unité logique du Texte (période révolutionnaire) ou de son affranchissement (périodes pré- et post-révolutionnaires), il importe de repérer le jeu d'une inféodation qui se réalise par le jeu d'une fiction opérante: l'unité textuelle, ainsi que ses corrélats de complétude et de cohérence, à défaut d'être posés, sont supposés et mis en oeuvre au plan du système global". ${ }^{18}$ S'agissant des mécanismes à l'œuvre dans l'interprétation judiciaire, Lenoble et Ost ont montré la tendance - ils parlent de "dérive mythologique" - du juge à faire "comme si" l'œuvre du législateur était le fruit de sa rationalité, d'abord en identifiant les contraintes discursives et juridiques affectant l'interprétation judiciaire, puis en en proposant un modèle descriptif théorique, en s'engageant enfin dans une analyse des fonctions de ce postulat.

Il faut constater la vigueur de la théorie qui veut que le travail jurisprudentiel consiste essentiellement en une application de la règle de droit aux faits qui sont présentés à l'instance judiciaire. Cette thèse repose sur trois représentations: le juge appliquerait le droit au fait étalé dans sa "réalité"; le langage juridique serait en adéquation avec la réalité qui lui est soumise et serait intelligible comme tel; l'absence de distorsion dans l'opération reliant le fait au droit. Or, du fait même de la transformation opérée sur le réel par son appréhension juridique et des glissements sémantiques qui affectent les termes de droit, c'est de manière tautologique que le droit fonctionne, la règle ne traitant que de ce qu'elle a au préalable assimilé et l'interprétation ne portant finalement que sur une substance prédéterminée par le langage juridique. La 
contextualisation de l'interprétation est indispensable, dès lors qu'on admet la polysémie des mots utilisés par le texte de la loi. ${ }^{19}$

Le syllogisme judiciaire - et donc la qualification judiciaire des faits - constitue la formalisation d'un processus d'application déductive du droit aux faits. Il ne s'agit pas d'un pur syllogisme, dans la mesure où il implique une dimension temporelle (contrairement au modèle logique). A la suite de ce que suggèrent Lenoble et Ost et comme le démontre parfaitement Jackson, ce syllogisme suppose une conception de la référence qui renvoie à une théorie de la vérité fondée sur la correspondance. Tel n'est pourtant pas, de toute évidence, le mode de fonctionnement de l'adjudication. Pour ne reprendre que le point de vue de la sémiotique discursive, le syllogisme judiciaire est un discours justificatif conférant un statut particulier à un discours adjudicateur. "Il ne peut vraiment y "référer", au sens de correspondre à la réalité externe du discours adjudicateur". ${ }^{20}$ Au modèle suranné du syllogisme judiciaire fondé sur la correspondance, Jackson oppose un modèle qui, au départ de la distinction entre l'adjudication et la justification, affirme le caractère narratif de la majeure et de la mineure et fonde la nature de leur relation en termes de cohérence. ${ }^{21}$ Le syllogisme judiciaire constitue ainsi un mode de justification des décisions et, de manière générale, le raisonnement juridique s'appuie sur des schèmes narratifs socialement construits. La décision, dans le processus d'adjudication, procède donc de la comparaison de l'unité narrative construite sur les faits de la cause avec le schéma narratif explicite ou implicite soustendant la règle juridique. "Plus celle-ci aura été abstraite de ses fondements narratifs, plus il y aura probablement des difficultés dans "l'application du droit au fait", malgré l'apparente clarté de la règle juridique et la possibilité apparente de lui subsumer les faits". ${ }^{22}$

Les faits et leur présentation sont eux-mêmes l'objet d'une construction passable d'une analyse en termes de narrativité. Le livre Charles Publications, 1988), 2-3.

22 Ibid., à 101. 
de Bennett et Feldmann ${ }^{23}$ en fait l'excellente démonstration. Pour ces auteurs, la construction de la réalité est, dans le processus judiciaire, une question de plausibilité narrative générale de l'histoire racontée, cette plausibilité étant elle-même fonction du stock de savoir social de l'instance décisionnaire (lui aussi très largement organisé en termes narratifs). La structure des histoires construites exerce un impact direct sur leur crédibilité. Cette structure est, par ailleurs, multiplement "biaisée" par les acteurs et par le fait qu'ils disposent ou non de routines cognitives leur permettant de présenter l'information dans des formes narratives admises et qu'ils partagent ou non des normes, des expériences et des présuppositions avec leurs interactants. ${ }^{24}$ Ceci étant, il convient de remarquer la focalisation exclusive des auteurs sur le niveau sémantique de l'opération de qualification, le niveau pragmatique restant pour l'essentiel occulté. ${ }^{25}$

On a déjà eu l'occasion d'évoquer, dans cette même perspective, le travail de Wagenaar, van Koppen et Crombag, ${ }^{26}$ qui proposent un modèle de la construction narrative du jugement judiciaire fondé sur l'ancrage des unités narratives dans le sens commun. ${ }^{27}$ Il n'est toutefois pas question de franchir le pas, comme le font Wagenaar, van Koppen et Crombag, du "fondement objectif" des vérités ancrées dans le sens commun. Sur ce point, comme sur nombre d'autres, le scepticisme de Jackson semble parfaitement justifié. 28

L'inventaire des éléments de preuve concernant l'affaire de la jeune fille de Ma`âdî, rédigé par le Président du Parquet du Tribunal plénier du Sud du Caire, constitue un document illustrant un des stades de l'opération juridique de qualification, à savoir la relation

23 W.L. Bennett and M.S. Feldmann, Reconstructing Reality in the Courtroom (New Brunswick: Rutgers University Press, 1981).

24 Ibid., à 171.

25 Jackson, supra n.20, à 70-76.

26 W.A. Wagenaar, P.J. van Koppen and H.M.F. Crombag, Anchored Narratives. The Psychology of Criminal Evidence (Hemel Hempstead: Harvester Wheatsheaf, 1993).

27 Cf. B. Dupret, "La définition juridique des appartenances", International Journal for the Semiotics of Law 30 (1997), 261-291.

28 B.S. Jackson, "Anchored narratives" and the interface of law, psychology and semiotics”, Legal and Criminological Psychology 1 (1996), 17-45. 
des faits de la cause et leur conformation pour la qualification et l'énoncé de la peine par le juge. Sans analyser le document de manière exhaustive, on relèvera plusieurs points intéressants. D'abord, la constitution de ce rapport en deux parties distinctes, la première se présentant comme le compte-rendu objectif des faits de la bouche de la femme victime de l'agression sexuelle, la seconde comme une liste de considérations émanant des autres parties présentes à cette agression et venant compléter le premier récit. Les faits sont donc établis à partir de l'exposé qu'en fait la victime principale. Ce qu'en rapporte la victime secondaire n'intervient qu'à titre subsidiaire, alors que ce qu'en disent les agresseurs se présente plutôt sous la forme d'aveux attestant le récit principal. Il apparaît très clairement que l'énoncé du récit ne se fait pas de manière libre, mais est au contraire structuré par les classifications juridiques dans lesquelles il faut l'insérer. On peut donc penser que ce que la victime principale a raconté, sur un mode probablement davantage "relationnel", selon l'expression de Conley et O'Barr, ${ }^{29}$ a été réorganisé directement (réécriture) ou indirectement (conduite de l'interrogatoire) par l'agent du Ministère public sur un mode "ruleoriented". 30 L'examen des considérations additionnelles des autres acteurs de l'incident vient conforter ce point. Le procès criminel étant organisé de manière accusatoire, c'est la sanction du criminel et non la réparation ou compensation du dommage subi qui conditionne la présentation des faits et l'imputation des responsabilités. Dès lors, l'accent est mis sur l'implication subjective de chacun des accusés plus que sur le dommage subi par les victimes. On constate de ce fait l'importance de l'aveu par chacun des protagonistes de son rôle personnel dans le crime: les cinq premiers accusés avouent "s'être couchés sur la victime", tandis que le sixième n'a fait que leur prêter assistance en connaissance de cause. De la même façon, l'intention d'agir criminellement transformant l'étendue de la sanction, on relève l'importance de la mention des circonstances ayant précédé l'accomplissement du crime: les cinq premiers accusés avaient décidé "d'enlever n'importe quelle femme qu'ils rencontreraient et de la

29 J.M. Conley and W.M. O‘Barr, Rules Versus Relationships. The Ethnography of Legal Discourse (Chicago and London: The University of Chicago Press, 1990). 
violer". Il en va de même de l'âge des protagonistes, qui lui aussi modifie la qualification et donc la sanction: la victime principale avait 17 ans (elle était donc mineure) et le rapport médical indique que "l'accusé Ashraf Hasan Jamîl a(vait) plus de 18 ans" (il était donc majeur). On peut également penser qu'alors que la nature profonde du crime ne s'en trouve pas modifiée, la triple qualification des faits (enlèvement et séquestration, vol à main armée, viol) permet d'imputer des circonstances aggravantes à la responsabilité des accusés et de dépasser le plafond légal prévu pour le viol.

Le juge dispose, sur la base de ce document, de la narration des faits et des responsabilités. C'est à lui que revient alors la charge de qualifier. Il ressort très clairement de ce qu'on vient de dire que cette qualification est préjugée, dès lors que l'organisation du récit est fonction des classifications juridiques auxquelles il convient de rapporter l'affaire. Ces classifications sont elles-mêmes des unités avant tout narratives témoignant, non pas de l'application d'un droit principiel à des faits établis objectivement, mais de narrations juridiques abstraites de leur contexte d'énonciation à des faits dont la narration est construite en fonction de la qualification à venir. A titre d'exemple, on peut rapidement s'attarder à la notion d'atteinte à la pudeur, telle que la doctrine et la Cour de cassation égyptiennes la définissent. L'atteinte à la pudeur ne correspond pas au viol, ce qui tendrait à éloigner l'exemple de notre cas d'application. Toutefois, l'atteinte à la pudeur occupe un statut intermédiaire sur l'échelle des crimes touchant à la moralité et, en ce sens, elle est d'autant plus illustrative du jeu de la narrativité qui traverse toute l'opération de qualification juridique. La doctrine donne de l'atteinte à la pudeur la définition suivante: il s'agit de "l'atteinte grave et délibérée à la pudeur de la victime par un acte portant atteinte à son corps et touchant généralement aux parties sexuelles", crime générique subdivisé en deux crimes distincts selon qu'il y a ou non usage de la force et de la menace. La sanction est stipulée par la loi; elle peut être aggravée si la victime est mineure ou incapable; elle varie selon qu'il y a ou non usage de la force et de la menace ou qu'il y a ou non préméditation; elle varie également selon que la victime est n'importe quelle personne ou bien le (la) descendant(e) de l'attenteur. La définition implique qu'il s'agisse: (1) d'un acte impliquant de saisir le corps de la victime; (2) d'une atteinte grave. Ceci distingue la catégorie "atteinte à la pudeur" de la catégorie "acte infamant" ( fi l 
fâdih). L'auteur tente ensuite de circonscrire le critère "parties sexuelles", tel que défini par la jurisprudence. En fonction de ce critère, ces situations sont considérées comme constitutives d'une atteinte à la pudeur. Cette liste, non exhaustive, est particulièrement illustrative du fait que la qualification n'est pas une application objective du droit aux faits, mais une constitution narrative du droit. Ainsi, étreindre la victime est une action attentatoire aux parties sexuelles, alors que le baiser sur les joues ne l'est pas. Cette distinction traduit une conception socialement et contextuellement identifiable de la sexualité (et de la moralité) qui, à son tour, marque la construction juridique de cette sexualité (et moralité), de l'atteinte qui peut lui être portée et de la sanction qui peut lui être attachée.

2. La typification des atteintes aux bonnes moeurs: confusion sémantique et distinction pragmatique

\subsection{Sens ordinaire et sens judiciaire: confusion sémantique}

Après avoir esquissé, dans un premier temps, l'opération psychologique par laquelle l'individu construit son univers de sens et d'action par voie de catégorisation et l'opération juridique visant à déterminer le droit applicable aux faits, je pense qu'il est à présent possible de souligner à quel point ces deux opérations ne procèdent pas de statuts radicalement différents, le profane d'une part, le professionnel de l'autre. Il me semble nécessaire de faire à ce stade quelques remarques sur la position du professionnel du droit par rapport à l'objet dont il est saisi. En un mot, je prétends qu'à l'image du sociologue et du savoir social, le juriste n'occupe pas une position totalement distincte et distanciée par rapport aux intrigues sociales qu'il doit investir de son savoir et au savoir social qui porte déjà sur ces intrigues. Dans cette démarche, nous suivons une perspective socio-phénoménologique et ethnométhodologique.

L'insertion dans des univers distincts justifie l'adoption de méthodes distinctes, non la détention d'un monopole du savoir et de l'objectivation du social dans le chef du professionnel. "La coupure épistémologique entre connaissance pratique et connaissance savante n'est pas de même nature que celle qui est habituellement admise par 
les sociologues".31 Le professionnel n'occupe pas de position de surplomb lui permettant de décrypter les règles auxquelles l'individu, considéré comme un "idiot culturel", ne ferait que se conformer. L'un et l'autre font de la "sociologie", utilisent des "ethnométhodes", typifient et catégorisent le monde, en un mot, produisent une visibilité du social permettant d'agir sur lui. Dans cette démarche, l'un et l'autre adoptent ce que Garfinkel a appelé, à la suite de Mannheim qui la réservait toutefois à la seule connaissance savante, une méthode documentaire d'interprétation. Ils recherchent le modèle (un pattern) homologue sous-jacent qui est à la fois désigné par l'occurrence et qui en même temps la désigne. Dans cette démarche toujours, profane et professionnel procèdent de manière indexicale et réflexive. L'indexicalité traduit le fait que, pour chaque individu, la signification du langage qu'il utilise dépend du contexte dans lequel ce langage apparaît, donc de ses conditions d'usage et d'énonciation. Ceci vaut aussi des gestes, des énoncés, des actions, etc. La réflexivité traduit, quant à elle, cette propriété qu'ont les activités de présupposer en même temps que de rendre observable la même chose. Elle constitue cet acte performatif qui, dans la description du social qu'il opère, produit ce social au-travers de "l'acquiescement motivé des personnes qui ont déjà ces attentes". ${ }^{32}$ C'est la pratique qui fait la règle, l'interaction qui dit le code, et non la règle qui conditionne la pratique ou le code qui détermine l'interaction: seule une actualisation constante permet à la règle ou au code d'exister. Ce qui distingue encore le profane du professionnel tient dès lors au contexte de leurs performances respectives, à leur appartenance à des "communautés" différentes engagées dans des "pragmatiques" différentes.

Les catégorisations spontanées des relations sexuelles que des magistrats égyptiens m'ont proposées (cf. supra) peuvent à nouveau servir d'appui à cette affirmation qu'il n'y a pas de déconnexion radicale entre le juriste et le profane et, partant, entre les sens ordinaire et judiciaire du droit et de la justice. On relèvera avec intérêt, même si c'est sur une question étrangère à la problématique de la moralité, que ces magistrats ont aussi comme référence, en

31 A. Coulon, L'ethnométhodologie (Paris: PUF, 1987), 50.

32 H. Garfinkel, Studies in Ethnomethodology (Englewood Cliffs, NJ: Prentice Hall, 1997), 55. 
matière de vol, l'image du vol de nuit par effraction. ${ }^{33}$ Sur les questions d'atteinte à la moralité, on peut par ailleurs rappeler qu'à l'évidence, ces magistrats procèdent par rapport au paradigme des "relations sexuelles licites". L'usage constant, dans l'affaire de la jeune fille de Ma'âdî, de la notion de virginité, que ce soit dans les documents légaux ou dans les articles de presse, atteste aussi de l'importance prise dans la narration par un concept qui n'est pas strictement juridique. La préservation de l'hymen de la jeune fille constituait certes une question à résoudre pour attester du viol. Il n'en reste pas moins que l'insistance sur sa virginité ne peut être limitée à une simple considération technique. Pourtant, ce type de considérations organisent la catégorisation des protagonistes et les droits et devoirs qui s'y attachent. On touche ici à l'organisation linguistique, contextuelle et indexicale du sens. Pour l'instant, on relèvera simplement que cette insistance n'est pas le fait exclusif de la presse: Parquet et juge s'appuient également sur une organisation narrative dichotomique opposant victime et agresseurs, "la jeune fille vierge" et "les six loups".

Cet entrecroisement des sens professionnel et ordinaire du droit et de la justice se retrouve aussi dans les articles de presse mettant en parallèle les dispositions du droit positif et les règles attribuées à la sharî'a islamique en matière de viol. J'ai déjà eu l'occasion de montrer que, s'agissant de la sharî $a$, les magistrats égyptiens semblent tributaires d'une "information sociale" qui conditionne leur propre perception métaphysique de la religion, de sa place dans la société que le droit dont ils sont porteurs prétend organiser et, dès lors, du champ des "possibles" et "impossibles" dans lequel inexorablement ils sont situés. Le professionnel du droit se trouve ainsi au centre d'une tension entre dynamiques normatives divergentes: celle de la science du droit positif et celle de la logique de la morale prédominante, elle-même traversée de courants multiples correspondant à la multiplicité des standards de vie. ${ }^{34} \mathrm{Je}$ mentionnerai simplement, dans cette perspective, qu'un professeur de droit traitant du viol du double point de vue de la sharî $a$ et du droit

33 Comparer avec Jackson, supra nn.4 et 28.

34 B. Dupret, "La sharî‘a comme référent législatif. Du droit positif à l'anthropologie du droit", Revue Interdisciplinaire d'Etudes Juridiques 34 (1995), 99-153. 
positif, introduit et conclut son article sur l'apologie des vertus et mérites du mariage et sur la nécessité de renforcer la sanction de l'atteinte portée à ce cadre légal qui est seul acceptable pour les relations sexuelles. Il ressort de cette présentation que la moralité sexuelle s'articule très clairement, dans sa double dimension professionnelle et profane, autour de la notion d'exclusivité maritale des relations sexuelles, le viol ne constituant de la sorte que la contradiction de cet idéal aggravée par l'usage de la contrainte.

\subsection{Sens ordinaire et sens judiciaire: distinction pragmatique}

Si l'on accepte la réduction de la fracture entre profanes et professionnels que la distinction des opérations cognitives de catégorisation et de qualification semblait avoir introduite, on devrait converger sur la nécessité de se doter d'un outil analytique permettant d'analyser d'un même mouvement l'appréhension du donné juridique. C'est dans la notion procédurale de typification que nous pensons pouvoir trouver l'outil en question. Sans entrer dans le détail, nous rappellerons simplement que la notion de typification peut être envisagée dans une perspective proche de celle proposée par la psychologie cognitive, avec ce que cela suppose comme risque de conception exclusivement descriptive de la référence, ou, pour tenter de parer ce risque, dans une perspective plus sociophénoménologique où elle prend la forme d'une opération antéprédicative qui s'apparente davantage à une formule d'opérations possibles qu'à un jugement de proximité relative. ${ }^{35}$

La typification ne consiste pas en une simple opération prédicative rapportant une situation à un paradigme, mais qu'elle opère de manière anté-prédicative, en-deçà donc de toute attribution intentionnelle de caractéristiques spécifiques à l'objet du jugement.

35 Cf. Jackson, supra nn.4 et 28, ainsi que "Towards a semiotic model of professional practice, with some narrative reflections on the criminal process", International Journal of the Legal Profession 1/1 (1994), 5579; cf. Quéré, supra n.11; A. Coulon, "Alfred Schutz", in K.M. Van Meter (sous la dir.), La Sociologie (Paris: Larousse, 1994); F.-A Isambert, "Alfred Schutz entre Weber et Husserl", Revue française de Sociologie 30/2 (1989), 299-319; D. Cefa•, "Type, typicalité, typification. La perspective phénoménologique", in L'enquête sur les catégories, supra n.11, pp.105-128. 
Ce stade anté-prédicatif est celui d'une pure réceptivité. ${ }^{36}$ Ce n'est qu'ensuite, à un niveau de justification sans doute, qu'intervient un jugement prédicatif faisant passer d'une typicalité factuelle à une typicalité générique qui, elle, suppose une activité réfléchie consistant à utiliser un concept pour déterminer un objet. En ce sens, la typicalité des jugements de catégorisation n'est qu'une thématisation de la typicalité engagée dans la pure réceptivité de l'expérience. ${ }^{37}$ Ceci explique que les types ne soient pas, a priori du moins, des modèles servant de base à la comparaison, mais qu'ils soient d'abord des structures de pertinence, 1 "'“index" pointant un problème". 38

Il reste toutefois que, si les typifications propres aux jugements professionnel et profane ne se distinguent pas par leur appui sur des stocks d'images - ces stocks d'images existant mais n'intervenant que par après - différenciées - quand bien même il y est fait référence, ces images procèdent souvent d'un stock commun, , elles n'en aboutissent pas moins à des résultats différents. Je serais tenté d'expliquer cela par la double insertion pragmatique de ces jugements: la pragmatique biographique des acteurs et la pragmatique structurelle de leur activité. Si donc on ne peut relever de spécificité sémantique des typifications ordinaire et judiciaire - parce que l'opération de typification n'est, d'une part, pas d'ordre principalement sémantique et que, par ailleurs, ce même niveau sémantique n'est pas différencié -, il faut par contre s'interroger sur sa spécification pragmatique. Celle-ci peut se situer dans la mise en scène de l'activité professionnelle (cf. Goffman), dans la narrativisation de la pragmatique professionnelle (cf. Jackson), dans l'organisation de l'activité pratique (cf. Sacks). Nous ne nous intéresserons qu'à ces deux derniers.

Jackson parle de narrativisation de la pragmatique. D'un point de vue sémiotique, la notion de "professionnel" est un attribut. ${ }^{39}$ Le syntagme narratif et les typifications narratives peuvent ainsi

36 On distingue deux caractéristiques: (1) il se constitue sur 1'arrière-plan d'un horizon de familiarité; (2) la détermination de 1'objet par son type est générale (précède la saisie de ses caractéristiques particulières) et approximative (mais peut être précisée de façon indéfinie).

Quéré, supra n.11, à 27.

38 A. Schutz, Collected Papers III (La Haye: Martinus Nijhof, 1966), 128.

39 Jackson, supra n.35. 
s'appliquer, non seulement au contenu d'un récit ou d'une action, mais aussi à l'acte même de communication de ce récit ou de cette action. Utilisant la métaphore de l'arbitre de football, Jackson montre que le professionalisme est véhiculé par le transfert de certaines modalités à l'intérieur de la pragmatique narrativisée de l'activité de juger plutôt que par une application déductive par le juge des règles du jeu aux faits qu'il rencontre sur un terrain quelconque. Ce transfert s'opère doublement: par la volonté d'"agir comme un bon juge"; par la communication du fait que l'obligation est apparemment satisfaite. Il s'agit donc du transfert, non pas seulement d'un savoir, mais aussi et surtout d'un savoir-faire, le professionnalisme devenant ainsi le moyen par lequel un client est persuadé que le savoir-faire qui lui est transmis est "compétent" (transfert d'une valeur sémiotique). Le propre du sens professionnel du droit ne tient donc pas tant à des différences dans les typifications du droit qu'à la modalisation de l'activité juridique. Adopter un cadre d'analyse faisant une part à la narrativisation de la pragmatique permet de restituer au comportement des participants à l'interaction juridique - relations client-avocat, jugement, cours, etc. - toute son importance, ${ }^{40}$ que ce soit dans la construction des faits ou dans la construction et l'application du droit. Ceci vaut en toutes circonstances, que les questions ne soient juridiquement pas controversées - comment la conviction du fait que ce n'est pas controversé a-t-elle été acquise ? qu'est-ce que "penser comme un juriste" ? quelle est la part du non institutionnel dans l'évaluation ? ou que l'affaire porte exclusivement sur un point de droit - des processus de perception, de stockage, de restitution, d'énonciation des faits et du droit sont engagés. ${ }^{41}$

Les sens ordinaire et judiciaire du droit et de la justice procèdent toutefois et avant tout d'activités pratiques. Les typifications dont ils font l'objet ne sont sans doute pas tant des opérations prédicatives que des "méthodes pour des activités pratiques", des "formules d'opérations possibles". ${ }^{42}$ C'est alors à l'organisation de ces activités pratiques qu'il faut d'abord s'intéresser. Tel est l'objet essentiel de l'œuvre de Harvey Sacks. Nous nous référons à la façon dont Rod

42 Quéré, supra n.11, à 37. 
Watson en rend compte. ${ }^{43}$ En affirmant le primat du pragmatique sur le sémantique, nous rejoignons en effet la préoccupation de Sacks pour l'ordre social et la façon dont on peut en rendre compte, dans la mesure où il s'agit d'une affaire familière et reconnaissable. L'activité ordinaire et judiciaire peut alors être considérée comme une activité sociale méthodique, procédurale, qui ne peut être envisagée que dans une perspective contextuelle ne voyant pas dans les catégories des dépôts de significations indépendants des circonstances de leur emploi, mais bien des ressources publiques et partagées utilisées pour donner un sens socialement standardisé aux situations. ${ }^{44}$ Ainsi, les catégories auxquelles correspond la paire relationnelle standardisée "professionnel/profane" (les deux catégories sont conçues comme "allant ensemble") sont-elles le lieu d'un ensemble de droits et d'obligations dont les éléments sont distribués selon un ordre lié intrinsèquement au contexte d'avantplan, l'activité juridique, et extrinsèquement au contexte contingent d'arrière-plan.

L'interrogatoire des délinquants mené par le Procureur permet d'illustrer cette question de la différenciation pragmatique. On peut d'abord observer un mélange de règles de procédure et de mécanismes conversationnels quotidiens, ce qui signifie que les environnements institutionnel et interactionnel de l'action opèrent simultanément. Alors que, dans la vie quotidienne, il existe de fortes contraintes sociales mettant un frein à la formulation d'accusations, il y a, dans le contexte judiciaire, de simples contraintes procédurales encadrant l'énoncé des accusations. ${ }^{45}$ On peut penser que ces contraintes sont en quelque sorte neutralisées par l'officialité d'une fonction, celle de procureur pour le cas qui nous concerne, qui institutionnalise et ritualise la construction et la présentation de l'accusation. Le récit du délinquant est également construit par le Procureur, de telle sorte qu'il anticipe les usages que le tribunal pourra en faire et qu'il devance les défenses possibles du délinquant.

43 R. Watson, "Catégories, séquentialité et ordre social. Un nouveau regard sur 1'oeuvre de Sacks", in L'enquête sur les catégories, supra n.11, pp.151-84.

44 Ibid., à 152-154.

45 M. Komter, "Accusations and defences in courtroom interaction", Discourse and Society 5/2 (1994), 165-187. 
Komter parle d'une forme d'hyper-accusation englobant les positions de transaction que le défenseur pourrait adopter de même que l'admission sélective qu'il pourrait opérer de ce qui lui est imputé. ${ }^{46}$ Cette anticipation est manifeste dans l'inventaire des éléments de preuve présenté par le Parquet. Comme on le faisait remarquer précédemment, l'intentionnalité des agresseurs est rendue manifeste, ce qui permet de précéder toute velléité de défense fondée sur l'impulsivité. La majorité d'un des prévenus est également établie, ce qui l'empêche d'alléguer de son âge pour échapper à la sanction prévue par la loi.

Le défenseur dispose de différents types de stratégie lui permettant d'échapper à sa responsabilité ou de l'atténuer. Il peut, d'abord, fournir des descriptions alternatives. La déposition d'un des agresseurs peut ainsi insister sur le rôle prépondérant d'un autre agresseur dans le crime qui a été commis, alors même que la déposition de ce dernier tend à davantage souligner le caractère collectif du crime. Une autre possibilité consiste à donner des motifs à l'action, des accounts dans le vocabulaire ethnométhodologique. Nous pouvons les appeler techniques de neutralisation. Dans notre affaire, le Procureur ne semble pas avoir laissé une place importante à l'usage de pareilles techniques qui pouvaient mener à l'atténuation du crime et, en conséquence, de l'accusation. Ceci étant, il faut relever l'argument d'un des agresseurs qui affirme avoir proposé au fiancé de la victime que la fille soit leur bien partagé (shirka), ce que le garçon aurait accepté. On peut penser que cet élément visait à atténuer la culpabilité des agresseurs en présentant l'affaire sous un jour de quasi-consentement des victimes. De même en va-t-il de cet autre motif avancé par un agresseur, qui tend à expliquer la décision de lancer une "chasse à la femme" par la déception qu'un autre des prévenus a connue quand il a vu la fille avec laquelle il flirtait partir avec un autre homme.

De manière générale, le défenseur semble répondre aux questions, non seulement en fonction de leur contenu factuel, mais aussi en fonction de ce qu'elles impliquent comme condamnation morale (blame-implicative nature). Ainsi, par exemple, en va-t-il de ce défenseur qui admet être rentré dans la pièce pour y violer la fille, mais qui explique que, parce que la fille lui a rappelé le caractère 
mauvais de son action, il a quitté la pièce sans avoir eu de relations avec elle.

Le défenseur doit aussi résoudre le dilemme consistant à fournir des informations sans porter atteinte à ses intérêts, à montrer sa volonté de coopérer tout en restant sur la défensive. Une admission partielle le rend coopératif, par exemple, tandis qu'une dénégation partielle lui permet de se défendre. Une autre stratégie de défense consiste, pour le défenseur, à estomper sa participation active aux événements. Il se fait ainsi jusqu'à un certain point disparaître comme agent de l'histoire. Les documents sur lesquels on a pu travailler laissent cependant apparaître une admission totale. L'explication tient, sans doute, au fait que l'enquête du Parquet fait suite à celle de la police. Il n'est pas rare, dans ce contexte, que les prévenus répètent un scénario construit par les inspecteurs de police et répété dans leurs locaux.

Si le travail du Procureur est officiellement d'examiner ce qui s'est objectivement passé et quelles sont en conséquence les responsabilités du défenseur, il se révèle également préoccupé de la culpabilité morale de ce dernier. Il n'est toutefois pas possible de relever, dans notre affaire, de formulation explicitement morale dans l'interrogatoire des prévenus par le Parquet. Ce type de propos doit probablement plus facilement apparaître à l'occasion des audiences des tribunaux et, plus particulièrement, au cours des interrogatoires et contre-interrogatoires de la Cour d'assises. Ceci dit, on notera qu'alors même qu'il recherche les faits, le Procureur dévoile souvent une position davantage morale. Il demande ainsi au défenseur, par exemple, d'évaluer ses actes. Ou il demande au défenseur de répéter ses aveux, ce qui lui permet de souligner les actes qu'il avoue et de lui faire admettre leur caractère mauvais. Ceci apparaît très clairement, dans l'affaire qui nous sert de support, à la lecture de l'interrogatoire du Parquet.

On notera enfin que le défenseur, pour sa part, ne cherche généralement pas à contester la moralité qui est en jeu; il tente juste de diminuer ce que cela pourrait inférer sur sa responsabilité morale. Aussi bien le Procureur que le défenseur semblent attachés à restaurer l'ordre moral: le Procureur en faisant en sorte que la conformité morale du défenseur soit exprimée, le défenseur en confirmant la moralité dominante tout en évitant les conclusions corollaires qui pourraient être tirées sur sa propre moralité. Nous pouvons observer 
ici une espèce de solidarité sans consensus: solidarité sur la moralité dominante dont aussi bien le Procureur que le défenseur disposent dans leur savoir social, sans consensus sur la mise en scène concrète de cette moralité dans l'affaire qui les met aux prises. 